\title{
Philosophiques
}

\section{Linéaments d'une phénoménologie des passions chez Ricoeur}

\section{Jean-Sébastien Hardy}

Volume 41, numéro 2, automne 2014

Centenaire de la naissance de Paul Ricoeur (1913-2013)

URI : https://id.erudit.org/iderudit/1027221ar

DOI : https://doi.org/10.7202/1027221ar

Aller au sommaire du numéro

Éditeur(s)

Société de philosophie du Québec

ISSN

0316-2923 (imprimé)

1492-1391 (numérique)

Découvrir la revue

Citer cet article

Hardy, J.-S. (2014). Linéaments d’une phénoménologie des passions chez Ricoeur. Philosophiques, 41(2), 313-332. https://doi.org/10.7202/1027221ar

\section{Résumé de l'article}

Cet article se donne pour tâche de reconstruire le projet d'une phénoménologie des passions formulé par Ricoeur au début des années 50 , projet pourtant délaissé ensuite. Après avoir explicité les motifs de cet abandon en les rapportant à la position du jeune Ricoeur face à la phénoménologie husserlienne, nous chercherons à montrer que ce dernier fournit néanmoins une définition proprement phénoménologique de l'affectivité passionnelle, qui ressaisit les passions selon leur intentionnalité spécifique et leur renvoi systématique à des passions originaires. Sur cette base, il s'agira enfin de mettre en lumière la relecture ricoeurienne de l'anthropologie kantienne des passions, qui culmine dans la thèse d'une fonction constituante des passions fondamentales (avoir, pouvoir et valoir) dans le rapport au monde vécu. 


\title{
Linéaments d'une phénoménologie des passions chez Ricœur
}

\author{
JEAN-SÉBASTIEN HARDY \\ Université-Laval/Université Paris-Sorbonne (Paris IV) \\ jean-sebastien.hardy@hotmail.com
}

\begin{abstract}
RÉSUMÉ. - Cet article se donne pour tâche de reconstruire le projet d'une phénoménologie des passions formulé par Ricœur au début des années 50, projet pourtant délaissé ensuite. Après avoir explicité les motifs de cet abandon en les rapportant à la position du jeune Ricœur face à la phénoménologie husserlienne, nous chercherons à montrer que ce dernier fournit néanmoins une définition proprement phénoménologique de l'affectivité passionnelle, qui ressaisit les passions selon leur intentionnalité spécifique et leur renvoi systématique à des passions originaires. Sur cette base, il s'agira enfin de mettre en lumière la relecture ricœurienne de l'anthropologie kantienne des passions, qui culmine dans la thèse d'une fonction constituante des passions fondamentales (avoir, pouvoir et valoir) dans le rapport au monde vécu.
\end{abstract}

\begin{abstract}
The aim of this paper is to reconstitute Ricœur's early project of a phenomenology of the passions. After having examined the motives which led Ricœur to leave this project behind, we will try to show that Ricœur nonetheless sketched out a rigorously phenomenological conception of the world of passions, by describing their specific intentionality as well as their systematic reference to the three fundamental passions of possession, power and honor. On this basis, the last scope of this paper will be to shed light on Ricœur's account of Kant's anthropology of passion, which leads to the idea that the fundamental passions are involved in the subjective and intersubjective constitution of the lifeworld.
\end{abstract}

\section{L'interprétation ontologique fondamentale de l'affectif en général n'a pratiquement pas pu faire un pas en avant significatif depuis Aristote ${ }^{1}$.}

L'histoire de la philosophie est le plus souvent conçue comme étant celle des doctrines philosophiques. Il y aurait pourtant lieu de considérer avec autant d'égards l'histoire de l'architectonique de la philosophie, c'est-à-dire celle de la mise en ordre et de la forme d'exposition (dialogue, traité, essai, etc.) auxquelles sont assignées les différentes matières directrices et annexes de la pensée philosophique. Jusqu'au siècle dernier, une pensée philosophique digne de ce nom ne pouvait manquer de donner lieu à une philosophie du droit, à une philosophie de l'histoire ainsi qu'à une esthétique, et l'idée d'une philosophie de la nature n'entraînait pas - jusqu'à l'Opus posthumum de Kant du moins - le sourire retenu d'une dérision faussement secrète. Parmi

1. Martin Heidegger, Sein und Zeit, Tübingen, Max Niemeyer, I967, p. I39. 
ces traditions déchues du système et de l'écriture de la philosophie, qui chacune à sa façon "redonnait prix aux choses » selon une expression de Montaigne, celle des traités des passions est des plus notoires tant un tel objet parait aujourd'hui étranger au déploiement authentique et rigoureux de la méditation philosophique. Qu'on en prenne pour exemple la Rhétorique d'Aristote, le Traité des passions de l'âme de Descartes, l'Éthique de Spinoza ou encore l'Anthropologie de Kant, l'analyse de l'affectivité passionnelle semble pourtant avoir de tout temps constitué un moment essentiel et vivant de l'invention philosophique.

Il serait présomptueux et inconvenant de chercher à rendre raison en quelques lignes de la désuétude de la théorie des passions au regard de la philosophie contemporaine. Il faudrait alors semble-t-il mettre avant tout en lumière le fait que c'est la psychologie philosophique elle-même - qui inscrivait la réflexion sur les passions en continuité avec la philosophie pratique et même avec la théorie de la connaissance (rhétorique ou didactique) — qui s'est trouvée dépaysée et finalement dissoute dans l'idée moderne et physiciste de la méthode. Et pourtant, même la psychophysique cartésienne des passions parvenait encore à nommer et à décrire - certes en partie dans la langue de la scolastique chrétienne, mais néanmoins dans la chose même certaines des structures insignes des passions qui sont toujours intimement nôtres.

Dans ce contexte, il aurait été légitime d'attendre que la phénoménologie, en vertu de sa révolution anti-copernicienne et du mot d'ordre d'un retour aux choses mêmes, se ressaisisse de cette sphère originale et originaire de l'expérience humaine qu'est la vie passionnelle. Heidegger affirmait en ce sens dans Être et temps que «c'est un mérite de la recherche phénoménologique que d'avoir procuré une vue plus dégagée sur ces phénomènes [que sont les affects et sentiments] ${ }^{2}$ », et Sartre s'enthousiasmait dans les années trente de ce que la percée de la philosophie husserlienne «a fait la place nette pour un nouveau traité des passions ${ }^{3} »$. Il est toutefois à première vue difficile de trouver dans la phénoménologie husserlienne une réanimation de la psychologia rationalis, dont le sens et le contenu ont été perdus au XIX ${ }^{e}$ siècle avec le tournant empiriste et expérimental de la psychologie. En effet, la phénoménologie de Husserl contribuera à sa façon elle-même à la critique du concept traditionnel de psychè, non seulement selon sa substantialité, mais aussi selon ses déterminations innées, caractérielles, affectives, pathologiques, etc. S'il est besoin de le rappeler, la conscience que découvre l'hypothèse méthodique de la destruction du monde aux paragraphes 49 et 54 des Idées directrices est bel et bien une "conscience sans âme». Plus spécifiquement toutefois, non seulement ne trouvera-t-on aucune mention de

2. Ibid., p. I39.

3. Jean-Paul Sartre, Situations philosophiques. I938-1966, Paris, Gallimard, I990, p. I2. 
l'affectivité dans le cours sur la Psychologie phénoménologique de Husserl ${ }^{4}$, mais, dès I90I, il était clair pour Husserl, fidèle en cela à Brentano, que les vécus affectifs, en tant qu'actes fondés sur des actes de représentation sousjacents (perception, souvenir, jugement, etc.), désignent une couche de la vie du moi qui n'est pas à elle seule et en elle-même originaires

Avec Scheler, Heidegger et Henry, la phénoménologie post-husserlienne aura certes repris à charge le projet d'une conceptualisation proprement philosophique de la vie affective. Il faut toutefois dire de façon générale qu'il ne se trouve pas de traité phénoménologique des passions dans les contributions pourtant essentielles de ces auteurs. La vie proprement passionnelle n'y est jamais considérée comme un objet propre et autonome, saisi selon ses divisions internes et structurantes ${ }^{6}$. Plus fondamentalement, le concept de passion doit d'entrée de jeu apparaître comme étant tout à fait irréductible au Gefühl schelerien, à la Stimmung heideggérienne ainsi qu'à l'autoaffectivité henryenne ${ }^{7}$ : passions et affects relèvent de deux ordres distincts de l'affectivité, comme l'a établi pour une première fois de façon nette l'anthropologie kantienne de la faculté de désirer ${ }^{8}$. Outre leur caractère foncièrement intentionnel, les passions (jalousie, avarice, orgueil, etc.) peuvent être distinguées de façon liminaire des affects (joie, colère, peur, etc.) par une certaine durabilité spécifique, par le fait qu'elles ne sont ni nécessairement ni proprement senties et enfin par leur caractère de projet ${ }^{9}$. La passion semble en cela être triplement irréductible à la conception henryenne de l'immanence pure de l'affectivité et échapper au moins en partie à l'analytique heideggérienne des dispositions inauthentiques du Dasein en sa quotidienneté10.

4. Si Husserl procède à une reprise critique de la psychologie physiologique des kinesthèses dans le cadre de sa phénoménologie de la perception (voir Chose et espace. Leçons de I907, Paris, PUF, I989), il ne conservera de la psychologie de l'affectivité que les concepts d'instinct et de pulsion, sans toutefois jamais les soumettre à une critique systématique, à moins d'interpréter le concept d'Erfüllung dans l'optique de la satisfaction d'un désir.

5. «Des intentions affectives ont pour base des intentions de représentations ou de jugements, etc.» (Edmund Husserl, Recherches logiques, tome 2, deuxième partie, Cinquième recherche [I90I], trad. de R. Schérer et al., Paris, PUF, I969, p. I69).

6. Heidegger remarque ainsi dans Être et temps qu' "au sein de la problématique de cette recherche, il n'est pas possible d'interpréter les divers modes de l'affection et les connexions de dérivation qui les relient» (M. Heidegger, Sein und Zeit, p. I38).

7. Voir le paragraphe sur les "Éléments d'une stratification de la vie émotionnelle» dans Max Scheler, Le formalisme en éthique et l'éthique matériale des valeurs... [1916], Paris, Gallimard, I955, p. 337-35I.

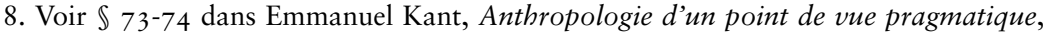
trad. d'A. Renaut, Paris, Flammarion, I993.

9. À cet égard, Kant disait d'ailleurs qu'elle est pour le sujet une "maxime d'action" prescrite à la faculté de désirer par l'inclination (Neigung) et qu'en cela elle a un "vernis de raison ». Voir E. Kant, Anthropologie, p. 237 et 242.

10. Quant à savoir si la phénoménologie du christianisme primitif (GA 60/I) et l'interprétation de la Rhétorique (GA I 8) menées par Heidegger seraient à même de contribuer de façon essentielle à une phénoménologie des passions, notamment grâce à la «destruction » de 
Il persisterait néanmoins une exception à cette proposition générale qui voudrait que la phénoménologie n'ait pas su livrer d'élucidation fondamentale et systématique de la vie passionnelle, ni même énoncer le programme explicite d'une phénoménologie des passions. En effet, les réflexions que mène Ricœur dans et autour des deux premiers volets de sa Philosophie de la volonté tracent de façon claire les contours d'une phénoménologie de l'affectivité passionnelle, en jetant du reste un premier éclairage sur ses données et problèmes fondamentaux. L'expression même de "phénoménologie des passions " apparaît en toutes lettres dans une communication de I95 I intitulée "Méthode et tâche d'une phénoménologie de la volonté», dans laquelle Ricœur revient de façon synthétique sur le projet global initié par sa thèse sur le volontaire et l'involontaire publiée l'année précédente ${ }^{11}$. Le mouvement qui anime et unifie les différents jalons de sa philosophie de la volonté est alors présenté comme celui du passage d'une phénoménologie de la volonté pure (qui correspond au premier tome de la trilogie) à une phénoménologie dite "ontologique», c'est-à-dire consacrée au moment négatif de la volonté (qui correspond aux deuxième et troisième tomes). En d'autres termes, en raison de la grandeur et de la misère de la liberté humaine, une analyse du sujet faillible et éventuellement coupable doit succéder à l'analyse initiale et préalable du sujet purement capable: "l'exégèse de ce moment négatif consiste pour une bonne part dans une phénoménologie des passions ${ }^{12}$ ».

Une dizaine d'années s'écoule pourtant entre les deux versants de la Philosophie de la volonté, si bien que cette deuxième partie annoncée ne verra finalement jamais le jour en tant que telle: à la phénoménologie des passions promise se sera substituée une "herméneutique des passions ${ }^{13}$. Le lecteur avisé retrouvera néanmoins sans difficulté, tant dans le quatrième chapitre de L'homme faillible que dans certains textes ultérieurs (notamment dans un article de 1959 intitulé «Le sentiment»), des indications précieuses en vue de l'édification d'une théorie résolument phénoménologique des passions. Avant de dégager et de regrouper les linéaments problématiques et positifs de la phénoménologie ricœurienne des passions, il importe toutefois de relever et de questionner les motifs qui conduisent Ricœur à renoncer à son projet inédit et prometteur.

la préconception psychologisante et anthropologisante de l'affectivité, et ce, en vue d'une compréhension ontologique de cette dernière, la question doit d'abord demeurer ouverte. Voir Christian Sommer, Heidegger, Aristote, Luther: les sources aristotéliciennes et néo-testamentaires $d$ 'Être et temps, Paris, PUF, 2005 et Theodore T. Kisiel, The Genesis of Heidegger's Being and Time, Berkeley, University of California Press, I993, p. 286-30I.

11. Paul Ricœur, "Méthode et tâche d'une phénoménologie de la volonté ", À l'école de la phénoménologie, Paris, Vrin, I986, p. 80.

12. Ibid., p. 8 o.

13. P. Ricœur, Philosophie de la volonté, 2. Finitude et culpabilité, Paris, Points, 2009, p. I 82 (désormais $P V$ 2). 


\section{Les motifs de l'abandon du projet ricœurien d'une phénoménologie des passions}

Au cours de la maturation et de la rédaction de sa philosophie des volontés libre et serve (unfreie Willen), Ricœur d'abord reporte, et ensuite délaisse l'idée d'une phénoménologie des passions. Il justifie sur plus d'une vingtaine de pages - réparties entre l'introduction générale du premier tome (intitulée "Questions de méthode ») et l'avant-propos du deuxième - l'impossibilité d'expliciter les passions au moyen d'une description phénoménologique directe, pure, et surtout transcendantale ${ }^{14}$.

Ricœur est au départ pourtant loin de remettre en cause la méthode phénoménologique dans son ensemble. Sa thèse sur Le volontaire et l'involontaire — rédigée en I 948 et parue deux ans plus tard, au même moment que sa traduction du premier tome des Idées directrices - est présentée comme une tentative de description eidétique des structures du vouloir décision, motivation, consentement, pouvoir, motion, etc. ${ }^{15}$. Ricœur s'y revendique d'une phénoménologie nettement plus husserlienne qu'herméneutique, mobilisant fréquemment et en divers contextes les gestes de la réduction phénoménologique, de l'analyse intentionnelle, de la variation imaginative ${ }^{16}$, et plus généralement de la description des essences.

Or l' «eidétique phénoménologique» des "structures fondamentales du volontaire et de l'involontaire» ne peut, selon Ricœur, avoir de validité que pour un vouloir conçu préalablement comme étant lui-même pur, c'està-dire conçu avant ou en deçà ${ }^{17}$ de sa contamination et de sa complication par l'incarnation, la faute et la transcendance, qui se révéleront comme autant de déterminations de la "situation fondamentale» de l'homme. Au même moment, Ricœur affirmera pourtant que les passions que sont "l'ambition, la haine, etc. sont la volonté même, [certes] sous sa forme aliénée ${ }^{18}$ » et non sous sa forme pure, ou innocente. Ricœur défend par conséquent de façon patiente, mais par moments embarrassée, la thèse selon laquelle la vie

14. Le sens et la légitimité de ce procédé d'abstraction a constitué un foyer privilégié de la réception de la philosophie de la volonté de Ricœur. Voir à cet effet: Frans R. Vansina, «La problématique épochale chez P. Ricœur et l'existentialisme ", Revue philosophique de Louvain, 70/8, I972, p. 587-619.

15. Voir P. Ricœur, Philosophie de la volonté, I. Le volontaire et l'involontaire, Paris, Points, 2009, p. 20-25 (désormais PV I). Ricœur confiera d'ailleurs plus tard qu'il concevait ce travail comme un prolongement de la phénoménologie de la perception de Merleau-Ponty pour ce qui est de la volonté et de l'agir. Voir P. Ricœur, "Herméneutique et symbolisme ", Le conflit des interprétations. Essais d'herméneutique, Paris, Seuil, I969, p. I8.

16. Voir notamment $P V$ 2, p. I 59 et I64.

17. Comme nous le verrons, Ricœur n'est pas tout à fait au clair avec le statut opératoire, fictif, ou généalogique que revêt l'exemple initial d'une volonté pure, avec tout l'imaginaire de l'innocence qui s'y trouve impliqué.

18. $P V$ I, p. 39 et 4 I. Italique ajouté par l'auteur. 
passionnelle s'excepterait du champ de la méthode et même du voir phénoménologiques.

Cette abstraction ou «mise entre parenthèses » (expression que Ricœur prend alors en un sens non technique) des passions et de la faute dans la description initiale de la volonté, annoncée par Ricœur dès la toute première page du Volontaire et de l'involontaire, nous livre en réalité deux indices significatifs, l'un quant à la précompréhension de la passion qui s'y joue, l'autre quant à la position du jeune Ricour face à la méthode phénoménologique.

L'abstraction des passions de l'analyse de la volonté pure nous enseigne d'abord quelque chose au sujet du pré-concept ricœurien de la passion, c'est-à-dire des déterminations traditionnelles de la passion que Ricœur admet avant toute analyse, et qu'il sera par la suite conduit à défendre ou à réviser. Bien que Ricœur exprime ses réserves quant à l'approche traditionnellement moraliste (tant au sens philosophique que littéraire) des passions ${ }^{19}$, il continue néanmoins à concevoir celles-ci, selon son propre aveu, du point de vue de ce qu'il appelle le "pathétique de la misère ». La passion concernerait l'homme dans la finitude de son vouloir, toujours guetté par la possibilité existentielle d'une perversion. Contre le "préjugé logiciste de Husserl ", dont la philosophie n'aurait "jamais pris au sérieux mon existence comme corps ${ }^{20} »-$ Ricœur introduit pourtant en France en 1952 les Idées II! ${ }^{21}$ - le domaine des passions échapperait notamment de la sorte à la discursivité primordiale du vécu:

Nous ferons toujours abstraction ici de ce brouillage des passions; nous nous sommes déjà expliqué sur cette méthode d'abstraction dans l'introduction; il nous faut apprendre le monde des passions par une autre méthode que l'approfondissement existentiel d'une eidétique: par la vie quotidienne, le roman, le théâtre, l'épopée; ce monde constitue un obscurcissement de la conscience qui ne se laisse pas comprendre comme le dialogue intelligible du volontaire et de l'involontaire ${ }^{22}$.

Ainsi, contrairement au "principe des principes » énoncé par Husserl, pour Ricœur le pouvoir de la description est un pouvoir restreint, qui connaît des limites autres que celles de ce qui se donne. Les passions seraient indéfectiblement marquées d'une empiricité telle que leur mise en lumière appartiendrait à un autre logos que celui phénoménologique. Les passions se rattachent aux yeux du jeune Ricœur à l'ordre du «mystère ${ }^{23}$ ", auquel

19. La faute est et doit être "sécularisée et incorporée aux éléments de l'existence humaine». Ibid., p. I45, note I I.

20. Ibid., p. 35 .

21. P. Ricœur, "Analyses et problèmes des Ideen II ", dans Revue de métaphysique et de morale, no 57, I952, p. 357-394.

22. $P V \mathrm{I}, \mathrm{p} .352$.

23. Voir ibid., p. 45 . 
participe d'ailleurs également l'incorporation de l'ego, fait qui excède toute constitution possible (le corps est dit «ingénérable $\left.{ }^{24} »\right)$. Et, à première vue, l'un ne va pas sans l'autre:

L'existence corporelle est un principe de confusion et d'indétermination [...]

Le projet est confus, le moi informe, parce que je suis embarrassé par l'obscurité de mes raisons, enfoncé dans cette passivité essentielle de l'existence qui procède du corps; le corps va devant comme "passion de l'âme» — ce mot étant pris en son sens philosophique radical: la passivité de l'existence reçue ${ }^{25}$.

Si les remarques du jeune Ricœur autour de l'empiricité irréductible du corps mériteraient d'être nuancées ${ }^{26}$, le plus étonnant demeure néanmoins le fait que, pour Ricœur - et c'est là une des thèses les plus novatrices de sa conception des passions -, les passions ne procèdent ni ne relèvent en rien $d u$ corps, "les passions procéd[ant] en effet du foyer même de la volonté et non du $\operatorname{corps}^{27} »$. Faute d'accepter sans plus la proposition vaguement romantique selon laquelle la passion est irrationnelle - ce contre quoi Ricœur luttera justement et habilement -, il importe alors, afin de mieux saisir le motif véritable de l'exclusion des passions du champ de la description des essences, d'examiner le rapport de Ricœur à la méthode phénoménologique elle-même.

Le refus de s'enquérir phénoménologiquement des modalités passionnelles de la volonté est en effet surtout révélateur de la position mitigée (quoique nuancée et consciente d'elle-même) de Ricœur face à la phénoménologie transcendantale au tournant du siècle dernier. Si d'une part Ricœur reconnaît ses droits - aussi délimités puissent-ils être — à la stricte description des essences, il conteste toutefois à diverses occasions l'omnipotence de l'ego transcendantal dans la constitution de la nature, de l'histoire et d'autrui $^{28}$, et ce bien avant les critiques célèbres formulées plus tard dans le Conflit des interprétations. Cette première prise de position de Ricœur face au tournant transcendantal de la phénoménologie est avant tout marquée et

24. Ibid., p. 35 et I 17 .

25. Ibid., p. 186.

26. Car si je ne peux par exemple agir au sein du monde des choses qu'en me mouvant et si je ne peux faire face à autrui qu'en me tenant devant lui, c'est donc bien que le corps propre a en son pouvoir de se mouvoir et de se présenter, et que réside là une condition transcendantale de la vie pratique et intersubjective: les diverses déterminations de la soi-disant facticité du corps renvoient en réalité à des déterminations transcendantales, c'est-à-dire qui sont proprement constituantes de toute expérience possible du monde.

27. Ibid., p. 40. Voir aussi ibid., p. 349.

28. Voir ce passage aux accents gadamériens: «La conviction qui circule en sourdine à travers les analyses les plus techniques est que la reprise sur soi de la conscience, lorsque celleci s'oppose à son corps et à toutes choses et tente de former cercle avec elle-même, est une perte d'être. L'acte du Cogito n'est pas un acte pur d'auto-position; il vit d'accueil et de dialogue avec ses propres conditions d'enracinement. L'acte du moi est en même temps participation » (ibid., p. 37). 
inspirée par la philosophie existentielle ${ }^{29}$; non par l'analytique existentiale de Heidegger, mais bien par l'existentialisme chrétien de Gabriel Marcel et celui de Jaspers, sur lesquels Ricœur avait tout récemment travaillé30. Résumant sa reprise de l'enjeu authentique de la philosophie husserlienne, Ricœur affirme: "Nous tenons à désolidariser la description pure, qui procède du serment de prendre les choses comme elles se donnent, d'une théorie de la constitution transcendantale du donné ${ }^{31}$. » La proposition originale de Ricœur est de faire valoir, non pas tant, comme plusieurs l'ont fait, l'intuitionnisme des Recherches logiques contre l'idéalisme postérieur, mais bien l'eidétique des Idées directrices contre l'égologie transcendantale ${ }^{32}$.

C'est finalement cette posture d'équilibriste qui commande la transformation de la méthode au sein du projet de la Philosophie de la volonté:

$[\mathrm{L}] \mathrm{a}$ considération de la faute et de ses ramifications passionnelles implique une refonte totale de la méthode. À partir d'un accident, une description eidétique n'est plus possible, mais seulement une description empirique. Le déchiffrage des passions exige que l'on apprenne l'homme par l'usage de la vie et les conversations ordinaires ${ }^{33}$.

Parce que l'égoïté des passions est toujours précédée par une appartenance à autrui, à l'histoire, à l'économie, etc., la description des passions ne peut pas prendre la forme d'un retour sur soi de la conscience phénoménologique (à la manière d'un récit de son auto-constitution), mais doit entreprendre plutôt le chemin long d'une «mythique des passions» et d'une «symbolique du mal» - moments déployés respectivement dans les volumes deuxième et troisième de la Philosophie de la volonté. En somme, les passions ne deviendraient intelligibles que par l'exégèse de certains mythes fondateurs ou particulièrement révélateurs - le péché originel, la chute, l'exil, le chaos, etc.

Dans la mesure où il s'agit pour nous d'esquisser une reconstruction de la phénoménologie des passions que Ricœur projetait aux débuts des

29. Voir ibid., p. 36, note 7. «Si c'est à Husserl que je devais la méthodologie désignée par le terme d'analyse eidétique, c'est à Gabriel Marcel que je devais la problématique d'un sujet à la fois incarné et capable de mettre à distance ses désirs et ses pouvoirs " (Paul Ricœur, Réflexion faite. Autobiographie intellectuelle, Paris, Esprit, I995, p. 24).

30. Voir P. Ricœur et Mikel Dufrenne, Karl Jaspers et la philosophie de l'existence, Paris, Seuil, I947 et Gabriel Marcel et Karl Jaspers. Philosophie du mystère et philosophie du paradoxe, Paris, Éd. du temps présent, I948. "En même temps que je souhaitais donner une sorte de complément à la Phénoménologie de la perception, j’espérais arbitrer en moi-même la confrontation entre Husserl et Gabriel Marcel » (P. Ricœur, "Herméneutique et symbolisme », p. I9).

31. $P V$ I, p. 77.

32. Ricœur réitère cette nuance dans "Méthode et tâche...», p. 66.

33. $P V$ I, p. 45. Sur cette dernière formulation cartésienne, voir «la reconnaissance comme identification» dans P. Ricœur, Parcours de la reconnaissance, Paris, Stock, 2004, p. $5 \mathrm{I}-52$. 
années 50 - à en croire le texte de "Méthode et tâche d'une phénoménologie de la volonté» —, la prétendue nécessité d'abstraire la passion d'une phénoménologie eidétique de la volonté (qui se bornera alors aux formes pures du projet, de la motion volontaire et du consentement) semble devoir être remise en cause et relativisée. Avant toute analyse, il ne se trouve en effet rien dans ce qu'est la passion qui interdirait a priori de la décrire phénoménologiquement - et que serait du reste une chose qui empêcherait d'emblée toute saisie phénoménologique d'elle-même, sinon une chose en laquelle plus rien ne se donne? Que d'une part les passions puissent bien relever d'une expérience dite "factuelle", cela ne les dépouille d'abord en rien d'une quelconque signification eidétique ${ }^{34}$. À cet effet, qu'y a-t-il à comprendre dans les mythes et les grandes passions tragiques, sinon précisément les relations d'essence qui s'y expriment et, à travers elles, la possibilité qu'elles aient été, qu'elles soient ou qu'elles puissent toujours être constitutives de nous-mêmes? Du reste, comme Ricœur s'est lui-même appliqué à le souligner dans sa traduction des Ideen I, la donation du fait implique d'emblée une donation d'essence ${ }^{35}$ et aucune description - même empirique - ne serait possible sans cette présence nécessaire de l'essence dans le "fait». Par exemple, comme mode d'être à la fois affectif et pratique, la cupidité (comme tendance accumulatrice) possède une certaine teneur pure de sens propre, qui la distingue notamment de l'avarice (comme tendance conservatrice).

Que d'autre part la passion puisse se saisir de l'ego au point de le réduire à son esclavage et ainsi d'aiguillonner l'entièreté de sa vie volitive, cela n'indique-t-il pas justement que la passion tient depuis toujours à la constitution la plus intime de l'égoïté même, et non seulement de la "personne" au sens transcendant? Non sans gêne, Ricœur concède à cet égard que «tenir en suspens les passions, c'est essayer d'abstraire les possibilités fondamentales de l'homme ${ }^{36}{ }^{3}$. L'ego, même transcendantal, n'est pas liquidé dès lors qu'on pense son exposition originaire à une affectivité qui le "surprend ", d'autant plus que Ricœur maintient, dans la lignée de Sénèque, qu'en un sens "nous nous donnons nos passions ${ }^{37}$ ", thèse essentielle qui

34. «Bien plus, le projet de Ricœur de mettre hors circuit la faute s'accorde parfaitement avec l'intuition eidétique husserlienne (die Wesensschaunng), adoptée par notre auteur. Celle-ci, en effet, met le fait entre parenthèses » (Vansina, "La problématique épochale chez P. Ricœur ", p. 593). L'interprétation de F. Vansina ne tient pas compte de l'interdépendance de la donation du fait et de la donation de l'essence que Ricœur souligne pourtant lui-même dans son commentaire infrapaginal des Idées directrices: "Tout fait comporte une essence [...] et toute essence comporte un champ d'individu » et "l'intuition de l'individu comporte la possibilité de convertir le regard du fait à l'essence» (Edmund Husserl, Idées directrices pour une phénoménologie, trad. et comment. de P. Ricœur, Paris, Gallimard, I950, p. I7, note I et p. I9, note 2).

35. Voir Husserl, Idées directrices..., $\mathbb{2}$ 2-3.

36. $P V \mathrm{I}, \mathrm{p} .40$.

37. Ibid., p. 35I. «Les passions offrent une illustration saisissante du "se faire souffrir soimême". En effet, à la différence des émotions, qui vont et viennent, des pulsions qui s'investissent dans des objets variables d'amour et de haine, les passions consistent en des investissements du 
mériterait une méditation phénoménologique approfondie. Pour finir, si comme le répète Ricœur «la passion est la volonté même ${ }^{38}$ », l'analyse des passions ne déborde peut-être pas tant le cadre de la phénoménologie transcendantale qu'elle s'y trouve appelée, voire requise, en ce que l'affectivité passionnelle, de par le caractère intentionnel que Ricœur lui reconnaît, peut prétendre participer à la constitution du rapport au monde, aux choses et surtout à autrui. Pour le dire autrement, il n'est pas impossible de penser, dans les termes de Heidegger cette fois, que «la tonalité affective [Stimmung] a à chaque fois déjà ouvert l'être-au-monde en tant que totalité [Ganzes], et c'est elle qui permet pour la première fois de se diriger-vers $[\text { quelque chose }]^{39}$ ".

La nécessité de limiter la description eidétique au seul champ de la volonté pure étant ainsi relativisée, il y a maintenant lieu de mettre en relief, avec tout le crédit qu'elles méritent, les réflexions proprement phénoménologiques quant au monde des passions que Ricœur mettra néanmoins de l'avant dans divers textes. Les plus cardinales de ces propositions se trouvent dans l'anthropologie de la faillibilité définie dans le premier livre du deuxième tome de Finitude et culpabilité (en particulier dans le paragraphe sur le $\theta u \mu o ́ s)$, en guise d'introduction à la symbolique du mal et à la mythique concrète, c'est-à-dire tout juste avant la première greffe de l'herméneutique sur la phénoménologie. Les énoncés directeurs de la phénoménologie des passions peuvent être rassemblés sous trois rubriques, soit a) l'intentionnalité, b) l'ordre et c) la réhabilitation éthique de la vie passionnelle.

\section{L'intentionnalité de la passion}

Les vrais objets sont à distance et les vrais sentiments sont profonds ${ }^{40}$.

L'analyse de la faillibilité qui constitue le premier livre de Finitude et culpabilité culmine en un examen du «rapport du passionné au passionnel», structure affective de la volonté qui s'avère être le "sol préalable de la faute ${ }^{41}$. La passion se révèle alors dotée d'un caractère foncièrement intentionnel, qui la distingue de la seule sensation, mais aussi de l'affect et du sentiment. Ricœur affirme ainsi que la passion est un «style d'ensemble qui a

désir dans des objets érigés en absolu » et «il est possible de concevoir une typologie du souffrir qui se réglerait sur celle de l'agir »; P. Ricœur, «La souffrance n'est pas la douleur », Psychiatrie française, numéro spécial, juin I992, respectivement p. 5 et 4.

38. PV I, p. 4I. Chez Descartes au contraire, la volonté exclut par définition la passion: «toute pensée qui ne vient que de l'âme est une volonté, toutes les autres sont des passions au sens large» (article I7).

39. M. Heidegger, Sein und Zeit, p. I37.

40. $P V$ 2, p. I34.

41. Ibid., p. I 82. 
son intentionnalité propre (celle de la jalousie, de l'ambition, de la haine cultivée, etc.), [et] où toutes les intentionnalités de fonctions partielles sont entraînées ${ }^{42}$ ». C'est dire qu'on ne peut pas comprendre une passion sans comprendre la donation de sens (Sinngebung) qui l'anime et au sein de laquelle l'objet apparaît (ou non) à la conscience qui vise affectivement celui-ci ${ }^{43}$. Plus exactement, la passion constitue pour Ricœur un style intentionnel, c'est-à-dire qu'elle donne et redonne sens à des intentionnalités subordonnées, ce qui signifie rien de moins que le sens même des différents actes fondamentaux du Je est intérieurement affecté par la passion. Ainsi, non seulement vouloir aura une signification différente pour l'ambitieux, le jaloux, l'avare, etc., mais se souvenir, se projeter, percevoir autrui, etc. recouvriront des fonctions différentes selon la passion qui anime le sujet (nostalgie, menace, défi, etc.).

Ricœur laisse entendre par ailleurs avec justesse, et ce contre la coutume de concevoir les passions comme un composé d'émotions, que l'émotion et l'affect possèdent leur sens par renvoi à la passion qui les sous-tend ${ }^{44}$. On pourrait montrer de cette façon que la colère du jaloux n'est pas la colère de l'avare, que le regret de l'ambitieux n'est pas le regret de l'amoureux, etc. La tendance interne à l'affect - si tant est qu'on puisse justement penser un affect quelconque sans référer d'avance au système passionnel qui lui donne sens, couleur et contenu — est originairement dirigée par le "projet » qui est celui du sujet passionné: la colère est tantôt frustration (comme privation), tantôt satisfaction (comme excuse), tantôt motivation (comme défi), etc. Il faut voir alors que les passions conditionnent l'apparaître même du sentiment, des choses et d'autrui, et ce dès avant leur surgissement manifeste.

Aux dires de Ricœur toutefois, l'intentionnalité ne caractérise pas seulement la passion, mais également le sentiment. Comment alors l'intentionnalité passionnelle se distingue-t-elle de l'intentionnalité sentimentale? En cela que le corrélat intentionnel, espéré ou craint, de la passion est toujours une représentation vide, c'est-à-dire que toute passion vise un «rien intentionnel », un objet qui paradoxalement ne peut pas être présent en chair de façon satisfaisante (erfüllende) : la soif du pouvoir vise ainsi une hégémonie sans frontière ni reste, la soif des honneurs vise une reconnaissance totale, la cupidité une richesse sans nombre, etc. Aussi le passionné ne peut-il se satisfaire que de simulacres ou de symboles de ce qu'il vise essentiellement. Parce

42. P. Ricœur, "Méthode et tâche...", p. 87.

43. Domenico Jervolino affirme ainsi à juste titre que "dans le sentiment, une intention et une affection coïncident dans le même vécu. Ici la réciprocité du sentir et du connaître empêche toute chute dans l'irrationalisme" (D. Jervolino, Paul Ricour. Une herméneutique de la condition humaine, Paris, Ellipses, 2002, p. 2I).

44. Voir P. Ricœur, "Méthode et tâche...", p. 87 et PV I, p. 40, où Ricœur insiste sur la nécessité de distinguer l'émotion de la passion. Ricœur remarque alors que "chez Descartes l'assimilation des passions à l'émotion est si complète que le traité des passions de l'âme est en réalité un traité des émotions fondamentales et de leurs complications passionnelles ». 
que l'objet ou la chose (Sache) de sa passion lui échappe toujours, la subjectivité passionnelle implique une opération proprement régulatrice de l'imagination, qui s'oriente sur l'Idée d'une donation effective de l'objet de la passion. En raison de cette démesure constitutive, la détermination fondamentale de la passion s'avère alors être sa vanité: la passion imagine comme tout ce qui est un rien, destiné à demeurer hors d'atteinte ${ }^{45}$.

Pour être vaine, la passion n'est pourtant pas sans efficace sur la constitution effective du soi. La formule consacrée par Ricœur pour caractériser le caractère foncièrement intentionnel de la passion est que celle-ci est une «manière d'être lié ou de se lier ${ }^{46}$ ». Cela doit certes signifier comme nous l'avons vu que la passion nous lie à un objet purement idéal, mais également que, ce faisant, elle nous lie le plus intimement qui soit à nous-mêmes, ce que l'on pourrait chercher à comprendre comme une figure de ce que Husserl nomme «intentionnalité longitudinale» (Längsinentionalität) ${ }^{47}$. En affirmant que «la passion, c'est la conscience qui se lie elle-même ${ }^{48}$ ", le jeune Ricœur fournit donc une piste de réflexion digne de considération quant au problème de l'unité et de l'identité du soi, qui viendra occuper de façon plus expresse l'œuvre ultérieure ${ }^{49}$.

\title{
Le problème de l'ordre des passions: le renvoi des passions aux modes insignes de l'objectivité
}

\author{
L'émiettement psychologique est sans fin; le principe \\ d'ordre ne peut venir que de l' "objet » ${ }^{50}$.
}

À diverses occasions, Ricœur critique de façon plus ou moins implicite la forme qu'a historiquement prise l'explicitation philosophique des passions,

45. «La passion est la puissance de la vanité; d'un côté, toute passion s'organise autour d'un rien intentionnel $[. .$.$] ; c'est ce rien spécifique, ce vain, qui habite soupçon, reproche,$ injure, grief, et fait de toute passion la poursuite du vent » (P. Ricœur, "Méthode et tâche...", p. 89. Voir aussi $P V$ I, p. 43 .

46. Voir notamment "Méthode et tâche...", p. 87. Italique ajouté par l'auteur.

47. E. Husserl, Vorlesungen zur Phänomenologie des inneren Zeitbewusstseins [1905], Halle, Niemeyer, I928, \39, p. I06-I09. L'intentionnalité longitudinale renvoie chez Husserl à l'auto-temporalisation de la conscience et se distingue en cela de l'intentionnalité transversale (Querintentionalität), qui renvoie à la temporalisation immanente des objets visés.

48. PV I, p. 348. Voir aussi ibid., p. 43.

49. Il serait intéressant de relire sous cet angle l'analyse de la temporalité du caractère, qui ne parviendrait pas à rendre compte de l'ipséité en tant que telle selon Ricœur dans Soimême comme un autre [1990]. Comprise phénoménologiquement, la passion n'échappe-t-elle pas pour sa part à cette psychologie de la mêmeté et ne vient-elle pas rendre compte d'une formation de l'ipséité qui ne passe pas par le détour de la narration, mais bien du rapport aux "objets» (pouvoir, estime, biens, etc.) qui me rapportent à autrui ? Il faudrait peut-être alors dire que la passion est toujours en elle-même une certaine narration silencieuse qui s'ignore. Voir P. Ricœur, Soi-même comme un autre, Paris, Seuil, I990, p. I43-I47.

50. $P V$ 2, p. 168. 
soit celle du traité, en laquelle le déploiement conceptuel de la vie passionnelle prend toujours les dehors d'un certain dénombrement de passions qui sont alors ou bien primitives, ou bien composées. Descartes affirme de cette façon à l'article 694 du Traité que l' "on peut facilement remarquer qu'il n'y en a que six qui soient telles [simples et primitives]; à savoir l'admiration, l'amour, la haine, le désir, la joie et la tristesse; et que toutes les autres sont composées de quelques-unes de ces six, ou bien en sont des espèces ». L'opposition de Ricœur à cette méthode d'approche de l'affectivité paraît tenir d'abord justement à la tendance analytique de Descartes et de saint Thomas en particulier ${ }^{51}$, qui s'empressent de reconduire l'ensemble des passions et sentiments à certains affects et appétits primordiaux, qui seraient du reste animaux et non spécifiquement humains ${ }^{52}$ : "Ces traités reposent sur cette idée que l'on peut dériver toutes les "affections" selon un ordre progressif du simple au complexe, en partant d'un petit nombre de passions simples, élémentaires ou principales ${ }^{53}$.» C'est l'une des raisons qui conduisent Ricœur à dire que les conceptions cartésienne et thomiste de la vie passionnelle relèvent en vérité d'une physique plutôt que d'une éthique des passions ${ }^{54}$.

Que cette critique sommaire du principe de la reconduction des passions complexes, dérivées et humaines à des passions simples, primitives et animales soit décisive ou non, elle pointe en direction d'un problème tout à fait fondamental pour Ricœur, à savoir celui de l'ordre des passions ${ }^{55}$. Car le véritable défaut formel du traité est de ne pas parvenir à poser de façon directe la question de la synthèse intérieure du monde des passions, c'est-à-dire ce qui les lie essentiellement entre elles (par subordination, exclusion mutuelle, etc.). Et à cet effet, la phénoménologie, aussi longtemps qu'elle demeure purement descriptive, ne paraît pas savoir y réussir davantage :

Il apparaît très vite que cette description s'éparpille dans des figures aberrantes indéfiniment multipliées; pour faire un monde, un cosmos, il manque aux passions un principe d'ordre [...] Comment dès lors une phénoménolo-logie des passions est-elle possible? A défaut d'un principe d'ordre, la phénoménologie saura-t-elle thématiser un principe de désordre?

51. Pour une critique de la primauté de l'irascible dans la complication de l'affectivité passionnelle, voir $P V$ 2, p. I $55^{-1} 58$.

52. "[La passion, en tant que maxime d'action,] est toujours associée dans le sujet à la raison, et à de simples animaux on ne peut attribuer de passions" (Kant, Anthropologie..., p. 237).

53. $P V$ 2, p. 137 .

54. "Mon dessein n'a pas été d'expliquer les passions en orateur, ni même en philosophe, mais seulement en physicien" (Descartes, Lettre à son éditeur, I4 août I649). Au livre III de l'Éthique, Spinoza propose de considérer «les actions et les appétits humains de même que s'il était question de lignes, de plans ou de corps».

55. Voir $P V$ 2, p. I74. 
Et, plus loin:

Une description [des passions] [est] menacée de s'éparpiller sans fin ${ }^{56}$.

Ricœur se met ainsi en quête du "principe original de la passion» et annonce dans Le volontaire et l'involontaire que ce sera l'un des objets du prochain volet de sa philosophie de la volonté que d'élucider ce principe positif de l'ordre des passions ${ }^{57}$.

C'est justement en lien à ce problème que Ricœur se rattachera ouvertement, dans Finitude et culpabilité, à l'Anthropologie de Kant, plus spécifiquement à sa tripartition des passions en quête de possession (Habsucht), quête de domination (Herrsucht) et quête d'honneur (Ehrsucht). Ricœur proposera alors une formulation proprement phénoménologique de la reconduction kantienne du monde des passions à ces trois «quêtes » inquiètes et fondamentales. Kant ne participe-t-il cependant pas par là à la façon classique d'éluder le problème de l'ordre immanent des passions? La thèse de Ricœur est que la radicalité des trois passions fondamentales ne tient plus cette fois à leur caractère élémentaire ou atomique, mais plutôt au fait que chacune d'entre elles serait fondée sur un domaine d'objets spécifique du monde commun. L'unité de la vie affective du sujet ne peut être alors reconquise en sa racine que par l'analyse préalable des objectivités vers laquelle elle est originairement, et pour des raisons constitutives, dirigée: la synthèse affective de la conscience subjective procède de la synthèse objective du monde $e^{58}$. Ce sera au moyen de l'analyse intentionnelle et eidétique de ces trois «objectivités» et d'un retour à l'intention originaire qui habite la passion en deçà de sa perversion que Ricœur répondra au problème de l'ordre des passions et jettera par le fait même un éclairage inédit sur l'affectivité passionnelle.

Ricœur s'applique en effet d'abord à montrer comment la division des passions dans l'anthropologie kantienne est fondée sur une triplicité du mode de donation du «monde interhumain ». Ainsi,

Il est possible de comprendre ce que serait une requête [...] d'avoir, de pouvoir, de valoir, en rapportant ces instances affectives successives à des dimensions correspondantes de l'objectivité; si notre théorie du sentiment vaut, les sentiments qui gravitent autour du pouvoir, de l'avoir, du valoir, doivent être corrélatifs d'une constitution de l'objectivité à un autre niveau que la chose simplement perçue $[\ldots]$ il faut ajouter à l'objectivité [naturelle] les dimensions

56. P. Ricœur, "Méthode et tâche...", p. I35-I36.

57. $P V$ I, p. 348 , note 8 I.

58. "Alors que l'imagination transcendantale se réduit tout entière à la synthèse intentionnelle, au projet de l'objet en face de nous, cette médiation se réfléchit en elle-même dans une requête affective indéfinie où s'atteste la fragilité de l'être humain. Il apparaît alors que le conflit tient à la constitution la plus originaire de l'homme; l'objet est synthèse, le moi est conflit. La dualité humaine se dépasse intentionnellement dans la synthèse de l'objet et s'intériorise affectivement dans le conflit de la subjectivité» ( $P V$ 2, p. I82-I83). 
économiques, politique et culturelle [...]. C’est donc le progrès de l'objectivité qui doit jalonner l'investigation de l'affectivité proprement humaine ${ }^{59}$.

Et, plus loin:

Il faut donc spécifier et articuler la relation du Soi à un autre Soi par le moyen de l'objectivité qui s'édifie sur les thèmes de l'avoir, du pouvoir et du valoir. Cette dernière remarque nous fournit, du même coup, un principe d'ordre ${ }^{60}$.

La «chose» (Sache) économique, la chose politique et l'estime servent alors de "guides " ${ }^{61}$, de fils conducteurs (Leitfaden), dans le dédale de la psychologie des passions. Les trois objets pathologiques généraux des diverses passions qu'identifie Kant, soit l'honneur, la domination et l'argent, renvoient ainsi à un objet authentique et plus originaire ${ }^{62}$, soit l'estime (et non la seule opinion), l'autorité (ou l'influence sur autrui en tant qu'autre libre) et le bien (en tant que chose que je possède comme corrélat d'une action, d'une protection, etc., possibles). L'analyse eidétique de chacun de ces objets permet alors tour à tour d'identifier les moments spécifiques de la constitution du monde commun autour desquels une passion déterminée s'articule ${ }^{63}$. Élucidant le sens de la chose économique, cette page de Finitude et culpabilité est particulièrement éloquente:

C'est en refluant de l'objet, comme objet économique, vers l'affectivité correspondante que l'on peut alors faire apparaître la nouveauté des "sentiments " qui s'attachent à l'avoir. [...] Le désir de l'objet économique est relatif à la disponibilité pour moi de cet objet; c'est la chose en tant que «disponible» qui suscite tout le cycle des sentiments relatifs à l'acquisition, à l'appropriation, à la possession, à la conservation [...] le moi est affecté par l'avoir, qui tient à moi et à quoi je tiens ${ }^{64}$.

Puisqu'un bien est une chose que je peux posséder en tant que je peux la perdre (et qu'en la perdant c'est une partie de mon pouvoir d'agir qui est perdu $)^{65}$, l'avarice apparaît selon sa rationalité immanente, soit le désir de maintenir mon pouvoir éventuel d'agir contenu en lui-même et non exposé à la possibilité de fait de sa liquidation.

Les passions apparaissent ainsi comme étant à l'origine orientées et ordonnées selon les divisions fondamentales du monde partagé, et elles ne sont toujours proprement passions qu'en ce qu'elles surinvestissent l'une de ces trois dimensions originaires au profit des autres. En conséquence, «il

59. Ibid., p. I60.

60. Ibid., p. I60-I6I. Italique ajouté par l'auteur.

61. Ibid., p. 174 .

62. Voir E. Kant, Anthropologie..., $\mathbb{\$} 84-85$.

63. Pour ces trois analyses successives, voir $P V$ 2, p. I6I-I74.

64. Ibid., p. I62. Italique ajouté par l'auteur.

65. Voir idem. 
faut creuser, forer sous les passions ${ }^{66}$ ", en un mot, retourner à l'intuition originaire du sens humain qui s'y joue, de leur «intention constituante ${ }^{67}$ ». La jalousie est ainsi un devenir pathologique du besoin de reconnaissance mutuelle, la cupidité un investissement excessif du besoin d'accumuler un avoir, etc.: "Nous ne comprenons, ensuite, les passions que comme écart, déviation, déchéance, à partir de ces requêtes originaires ${ }^{68}$."

On notera au passage que, dans la mesure où les passions kantiennes renvoient chacune à un milieu culturel et historique partagé, «d'emblée est contestée la validité d'un schéma indifféremment animal ou humain de l'affectivité69 ". Ricœur reprendra de cette façon à Kant l'idée selon laquelle toute passion est en son fond intersubjective ou, plus exactement, interhumaine $^{70}$. Kant affirmait en effet dans l'Anthropologie que «toutes les passions ne sont toujours que des désirs d'êtres humains envers des êtres humains, et non pas envers des choses ${ }^{71}$ ", ce qui est sans doute plus évident dans le cas des passions de l'honneur (orgueil, ambition, etc.), mais qui est tout aussi vrai dans le rapport passionné à la chose économique.

Il faut ajouter enfin qu'aux yeux de Ricœur les trois domaines de l'objectivité sont eux-mêmes ordonnés entre eux: le valoir est fondé sur le pouvoir qui est fondé sur l'avoir ${ }^{72}$, si bien que l'on semble devoir conclure de cet ordre quasi-dialectique de fondation que le monde interhumain est originairement économique. Cela dit, l'objectivité économique enveloppe elle-même l'intersubjectivité, en ceci que toute chose déposée dans le monde apprésente autrui et donc la possibilité d'un conflit des intérêts: la propriété suppose donc à son tour toujours la reconnaissance. Ainsi, même l'avare esseulé ne veut son argent que contre autrui: en tant qu'elle renvoie à une activité de conservation et de protection de ce qui fut eu ou mérité, aucune expérience

66. Ibid., p. I 55 .

67. Ibid., p. I69.

68. Ibid., p. 159.

69. Ibid., p. I 58 .

70. Idem.

71. E. Kant, Anthropologie..., $\mathbb{S} 80$, p. 239; idée reprise par Kant au $\mathbb{8}$ 82, p. 242.

72. Voir $P V$ 2, p. I73. Une description de l'envie pourrait sans doute jeter un éclairage sur les implications mutuelles des trois passions fondamentales. Voir P. Ricœur, "La souffrance n'est pas la douleur ", p. 5: "Je souffre de ne pas avoir ce que l'autre a, parce que c'est l'autre qui l'a, et pas moi. » De la même façon, les passions de l'argent, en tant que celui-ci est une puissance indéterminée d'avoir et de s'arroger, semblent toucher aussi au pouvoir. Il est intéressant que Ricœur considérera plus tard cette co-implication des trois passions fondamentales à partir d'une toute autre référence philosophique: "On connaît par leur nom les trois passions primitives qui ensemble caractérisent l'état de nature comme "guerre de tous contre tous". Ce sont la compétition, la défiance, la gloire. "La première pousse les hommes à attaquer pour le profit, la seconde pour la sécurité et la troisième pour la réputation” (Hobbes, Léviathan, p. 224). Il est remarquable qu'aucune de ces passions ne se conçoit sans une référence à l'autre!» (P. Ricœur, "La reconnaissance mutuelle», Parcours de la reconnaissance, p. 242). 
de pensée ne pourrait nous rendre l'image d'un avare qui soit solus ipse $e^{73}$. Il y aurait en ce sens une confusion ou plutôt une co-originarité des trois objectivités constituantes de l'humanité, et donc une co-originarité de ses trois passions fondamentales et fondatrices.

\section{Conclusion: la passion comme « requête d'humanité» et la question du caractère éthique de la passion}

Je pense en particulier qu'à partir d'une réflexion sur le volontaire et l'involontaire on passerait à une deuxième phase, à une phase éthique, lorsqu'on réintroduirait des éléments qui n'ont absolument pas paru dans mon analyse: je pense à l'analyse des passions ${ }^{74}$,

En lien avec cette intersubjectivité de la passion, il faut voir maintenant que, si de prime abord le sujet passionné paraît rester toujours au plus près de luimême, consommé qu'il est par un objet inaccessible et donc seulement imaginé, la "pneumatologie» ricœurienne rend bien manifeste que cette obnubilation recouvre en réalité un désir d'humanité qui cherche à se frayer par et malgré le rapport à l'objet. Et c'est par cette thèse d'une "requête d'humanité » qui formerait le noyau de sens de l'intention passionnelle que Ricœur assume jusqu'au bout et approfondit la thèse selon laquelle l'ordre des passions renvoie en dernière instance à celui d'objectités insignes. Possession, domination et valorisation représentent trois voies ou moments nécessaires de la constitution du soi au sens plénier ${ }^{75}$. Toujours en référence à l'anthropologie kantienne des passions, Ricœur affirme, dans une page importante de l'article de 1959 sur "Le sentiment»:

Les affections qui remplissent l'intervalle [entre le vivre et le penser] sont celles qui animent les passions les plus importantes de l'histoire et de la culture; non plus ces passions élémentaires des Traités des passions, mais au contraire les

73. Dans un texte intitulé "L'argent: d'un soupçon à l'autre », Ricœur montre que, économiquement neutre, l'argent n'est pas pour autant «moralement» neutre: «Le prix résulte d'une concurrence de convoitises appliquée à des biens rares offerts aux appétits de tous; dès lors la grandeur des biens acquis porte la marque de l'inassouvissement des désirs des autres. Un petit jeu cruel se cache ainsi dans le moindre échange, où ne semblait d'abord entrer en ligne de compte que la liberté de l'un de se dessaisir et la liberté de l'autre de choisir. L'exclusion d'un tiers invisible se dessine en pointillé derrière le geste simple d'acheter et de vendre » (P. Ricœur, "L'argent: d'un soupçon à l'autre ", L'Argent. Pour une réhabilitation morale, A. Spire (dir.), Paris, Éd. Autrement, I992, p. 6I).

74. P. Ricœur, "L'unité du volontaire et de l'involontaire», Bulletin de la Société française de philosophie, Séance du samedi 25 novembre I950, document disponible sur http:// www.fondsricœur.fr, p. 2 I.

75. «Il est remarquable que le Soi n'est jamais assuré: la triple requête dans laquelle il se cherche lui-même n'est jamais achevée» $(P V$ 2, p. I76). 
passions essentiellement interhumaines, sociales, culturelles, communautaires, que Kant plaçait sous le triple titre de passion d'avoir, passion de domination et passion d'honneur. Car derrière ces figures passionnelles aberrantes, voire bideuses et meurtrières, se tiennent des requêtes qui sont d'authentiques requêtes d'humanité: le moi y constitue sa différence - sa différence d'avec les choses et sa différence d'avec les autres moi; le moi se distingue par le mien, s'affirme par l'autorité, cherche sa valeur dans l'opinion d'autrui, dans l'approbation et dans l'estime. Je n'ai pas le temps de pousser bien loin cette esquisse, ni de retrouver la bonté originelle de ces trois requêtes, par-delà leurs expressions historiques mauvaises, ni de montrer les correspondances entre ces requêtes affectives d'avoir, de pouvoir et de valoir et la constitution de nouvelles couches d'objectivité d'objets de niveau humain, réalités économiques, réalités politiques, réalités culturelles ${ }^{76}$.

Cette rémanence, au sein de la passion, d'une requête authentique d'avoir, de pouvoir ou de valoir interdit par là définitivement que la théorie ricœurienne des passions retombe, contre ses intentions initiales, dans une condamnation morale ou moralisante des passions. Ricœur révèle même au contraire une genèse en partie transcendantale des passions, dont le caractère essentiel n'est plus la perversité, mais bien à l'inverse le fait qu'elles découlent de l'histoire même d'un soi qui cherche et doit chercher à avoir mainmise sur son monde environnant, à avoir de l'influence sur l'agir des autres et à être reconnu par autrui. Une "bonté originelle » traverse ainsi chacune des passions: "Le dévouement inquiet du passionné est comme l'innocence primordiale du passionnel et en même temps la fragilité essentielle d'où elle a procédé77. " Le cogito passionné n'est en dernière instance peut-être pas tant un cogito humilié qu'un cogito dévoué et dévoyé, épris et pris dans une interprétation objectivante et exclusive d'une quelconque de ses trois requêtes d'humanité.

Comme le remarque pertinemment Frans Vansina :

De l'enveloppement des passions et de la loi dans le mal il s'ensuit que leur abstraction entraîne aussi celle de l'éthique réelle. La raison en est que la description pure du volontaire et de l'involontaire, et, par là, de la liberté et des valeurs, ne fournit que l'ossature abstraite pour une morale concrète qui commence, en fait, par la considération des passions ${ }^{78}$.

Parce que toute passion est constituée sur la base d'un rapport du soi à une des dimensions constituantes du monde de la vie partagé, parce que toute passion engage la présence — ne serait-ce que virtuelle — d'un autre moi, la phénoménologie des passions du jeune Ricœur émerge en effet sur la question du caractère éthique de l'affectivité. Contre l'optique classique

76. P. Ricœur, «Le sentiment", À l'école de la phénoménologie, p. 27I. Italique ajouté par l'auteur.

77. $P V$ 2, p. I 82 .

78. F. Vansina, «La problématique épochale... », p. 589. Italique ajouté par l'auteur. 
d'une pré-compréhension pathologique des passions, la thèse de Ricœur est que toute passion est habitée d'une "requête plus originaire qu'elle». Entamant par là le projet d'une dépsychologisation et surtout d'une démoralisation de la passion, Ricœur est ainsi conduit à faire jouer Kant contre Kant, chez qui les passions représentaient encore autant de formes déchues de la sensibilité pratique, et même une "maladie de l'âme» (Krankheit des Gemüts):

Une anthropologie philosophique [plutôt que pragmatique] doit être plus exigeante; elle doit procéder à la restauration de l'originaire qui est à la racine du déchu $[. .$.$] il faut retrouver, derrière ce triple Sucht, la quête non plus folle et$ serve, mais constitutive de la praxis humaine et du Soi humain ${ }^{79}$.

L' "usage des passions", pour reprendre une expression cartésienne, enferme alors un enjeu proprement éthique - au sens large, et moralement neutre, de la constitution d'un monde commun, partagé et animé de certaines significations et valeurs fondamentales, telles que le pouvoir-faire, l'estime, la conservation, l'accumulation, etc., qui fonctionnent comme autant de motifs critiques et a priori de l'action au sein d'un monde où l'on doit avoir, valoir et pouvoir pour seulement être. Ainsi, les passions «concernent l'ethos humain dans son ensemble ${ }^{80}$ ». D'un point de vue phénoménologique, la réflexion éthique ne peut commencer de façon légitime et assurée qu'une fois qu'ont été explicitées les conditions sur la base desquelles une action est reconnue comme celle d'un sujet (reconnu comme tel, imputable, etc.) en un monde qui est à la fois partagé par d'autres sujets. À ce compte, les descriptions denses que mène Ricœur de l'avoir, du pouvoir et du valoir - mais ne devrions-nous pas y inclure la passion de savoir, avec ses formes passionnelles que sont l'encyclopédisme, l'ésotérisme, le dogmatisme, etc. ? - mettent précisément en lumière ces constituants primaires du monde éthique.

Il faudrait même aller plus loin et dire que la description des passions retrouve et même découvre l'imaginaire de l'innocence de la volonté auquel Ricœur croyait ne pouvoir accéder qu'en renonçant à considérer les passions. La phénoménologie des passions du jeune Ricœur culmine ainsi en une réhabilitation relative de la passion, dont on retrouve pour ainsi dire l'innocence dès lors qu'on en identifie les «intentions constituantes ${ }^{81}$ ».

Comme nous l'avons entrevu, nous pourrions relever tant chez Ricœur que chez Levinas et Henry (dans son Marx) des réflexions cruciales quant aux

79. $P V$ I, p. I58. Italique ajouté par l'auteur.

80. P. Ricœur, "Méthode et tâche...", p. 8I.

81. "Il faut même dire que ce que nous comprenons d'abord, ce sont les modalités primordiales du désir humain et constituantes à l'égard de l'humanité de l'homme [...] Cette compréhension de l'originaire d'abord, du déchu ensuite, à partir et par le moyen de l'originaire, requiert sans doute une espèce d'imagination, l'imagination de l'innocence, l'imagination d'un "règne" où les requêtes d'avoir, de pouvoir et de valoir ne seraient pas ce qu'elles sont en fait " (PV 2, p. I 59). 
"passions économiques », ces auteurs ayant dégagé les éléments constitutifs d'une économique fondamentale de la vie (production, travail, besoin, avoir, habiter, etc. $)^{82}$. Et cette légitimation de la passion sur la base de la requête authentique qui la fonde et en lequel le sujet peut éventuellement s'abîmer est tout autant perceptible dans ce passage admirable d'une conférence intitulée «Sympathie et respect. Phénoménologie et éthique de la seconde personne »:

Et même, peut-on dire, le jaloux perce avec plus de clairvoyance le décor de gesticulation d'autrui en direction du foyer d'intentions où se constitue son existence que le brave homme qui trouve tout le monde sympathique. La façon dont le jaloux épie les signes, les suspects, les confronte, ne constitue-telle pas une sorte de doute méthodique, impitoyable et douloureux, opposé à tous les jugements précités de la sympathie, à cette naïveté de la sympathie qui, spontanément, attache une pensée, un sentiment, une intention à une expression, à une conduite? N'est-ce pas la jalousie qui, mieux que la compassion, reporte et repousse autrui au-delà des signes qu'il donne de lui-même? Le jaloux n'atteste-t-il pas, par sa souffrance même, l'existence d'autrui, l'existence absolue et certaine d'un Soi étranger et inaccessible, qui se cache autant qu'il se montre dans le phénomène de lui-même? ${ }^{83}$

Il est désormais manifeste que Ricœur a finalement été conduit, malgré son refus initial d'entamer le projet d'une phénoménologie des passions, à poursuivre de façon cohérente, signifiante et novatrice certaines intuitions fondatrices d'une phénoménologie de l'affectivité passionnelle. En remarquant d'entrée de jeu que, pour le moi concret, "la passion est la volonté même ", Ricœur jette en effet les bases d'une description eidétique des passions fondée sur l'analyse préalable du monde interhumain, grâce à laquelle la passion apparaît finalement comme un "vouloir constituant» (fût-ce de façon empêchée ou pervertie), et ce, tant au niveau restreint de la psychè individuelle qu'à l'échelle du monde vécu et partagé, lui-même compris dans ses caractères historiques, culturels et économiques ${ }^{84}$. Se rattachant à la formule hégélienne selon laquelle rien de grand ne s'est fait dans le monde sans passion, Ricœur en viendra même à affirmer que celle-ci «anime le mouvement de l'histoire et, en jetant l'homme vers le mieux-être et le pouvoir, fonde l'économie et la politique ${ }^{85} »$ ! Le programme que nous lègue le jeune Ricœur, et qui gagnerait à se nourrir de l'œuvre ultérieure, est alors celui de mener sur deux fronts simultanément la description du monde et celle des passions, en vertu de leur co-originarité essentielle.

82. Voir aussi Jean Greisch, Paul Ricœur. L'itinéraire du sens, Grenoble, Million, 200I, $\$ 5$, c: «La nature conflictuelle de l'homme: les passions humaines fondamentales».

83. P. Ricœur, "Sympathie et respect. Phénoménologie et éthique de la seconde personne» [1954], À l'école de la phénoménologie, p. 272-273. Italique ajouté par l'auteur.

84. De façon exemplaire, Ricœur montre dans son article sur l'argent mentionné plus haut comment l'histoire du libéralisme est une histoire de la transformation des passions directrices du sujet économique.

85. P. Ricœur, "Méthode et tâche... », p. 89. 